\title{
The Effect of Chicken Spleen Transfer Factor on Intestinal Mucosa Immunity Barrier of Laying Hens
}

\author{
Yu J ${ }^{1 \#,}$ Ma $B^{2 \#, ~ L i ~ J ~}{ }^{1}$, Chen $Y^{1}$, Wang $Z^{1}$, Cao J ${ }^{1}$ and \\ Dong $Y^{1 *}$ \\ ${ }^{1}$ Key Laboratory of Precision Nutrition and Food Quality, \\ Ministry of Education, College of Veterinary Medicine, \\ China Agricultural University, Beijing, China \\ ${ }^{2}$ China Animal Husbandry Group, Beijing, China \\ "Contributed Equally to this Work \\ *Corresponding author: Yulan Dong, Key Laboratory \\ of Precision Nutrition and Food Quality, Ministry \\ of Education, College of Veterinary Medicine, China \\ Agricultural University, Beijing, 100193, China
}

Received: J uly 15, 2021; Accepted: J uly 31, 2021; Published: August 07, 2021

\begin{abstract}
Chicken spleen Transfer Factor (TF) is a low-molecular-weight lymphocyte extract composed of polypeptide and nucleotide. However, its role in regulating intestinal structure and function in laying hens has remained largely unknown.

100 one-day-old laying hens were randomly divided into five groups and administered with different doses of TF $(0.00$ [control], $0.05 \mathrm{~mL}, 0.10 \mathrm{~mL}$, $0.25 \mathrm{~mL}$ and $1.00 \mathrm{~mL})$. The results showed that the high dose of $\mathrm{TF}(1.00 \mathrm{~mL})$ improved the intestinal mucosa morphology and strengthened the digestive and absorption function. Furthermore, the histology analysis revealed an increase in the number of intraepithelial lymphocytes and goblet cells. Similarly, the results from ELISA demonstrated an increase in the content of IL-10 in the intestinal tract, while the content of TNF- $\alpha$ showed a decrease in this regard. The RT-PCR assay also demonstrated the upregulation of the relative mRNA expressions of Muc2, TLR-2, and TLR-4 genes. The intestinal antioxidant function was significantly enhanced. In conclusion, high-dose of TF can improve the intestinal mucosa morphology and structure, enhance digestion and absorption functions, enhance the intestinal mucosal barrier immune function and antioxidant function, and up-regulate Muc2, TLR-2 and TLR-4 gene relative expression.
\end{abstract}

Keywords: Chicken spleen transfer factor; Intestinal, immune; TLR, Antioxidant function

\section{Abbreviations}

TF: Transfer Factor; Muc2: Mucin 2; PAS: Periodic Acid-Schiff; IELs: Intra-Epithelial Lymphocyte; TLR: Toll like Receptor; IEC: Intestinal Epithelial Cell; VH: Villus Height; CD: Crypt Depth; SOD: Superoxide Dismutase; CAT: Catalase; GSH-PX: Glutathione Peroxidase

\section{Introduction}

Nowadays, rapid developments in biotechnology, biopharmaceuticals, peptides, and proteins have emerged with a host of new applications in diagnosis and therapy $[1,2]$. In contrast to small molecules, peptides and proteins have the advantages of high efficiency and selectivity, as well as low toxicity [3]. In addition to direct antibacterial activity, antimicrobial peptides also have immunomodulatory properties [4]. The antimicrobial peptide cecropin $\mathrm{AD}$ improves the immune status, as well as nitrogen and energy retention, thereby reducing intestinal pathogens in weaned piglets [5]. Other studies have shown that fungal-derived macromolecular substance includes a blend of yeast-derived nucleotides, inositol, and protein as immunomodulators to promote the pathogenic microbe control $[6,7]$.

Transfer Factors (TF) are low-molecular-weight lymphocyte extracts consisting of polypeptide and nucleotide which transmit the ability to express delayed-type hypersensitivity and cell-mediated immunity from high responder animals to low responder animals while the reverse is not true [8]. At present, since TF is a complex group composed of many low molecular weight proteins, the exact chemical properties and molecular mechanism of $\mathrm{TF}$ have not been clarified. Nevertheless, several studies have shown that TF is a potential immunotherapeutic agent.

In a recently conducted study, TF has been used successfully as adjuvant or primary therapy for various immune dysfunctions. Moreover, several clinical and experimental studies have demonstrated the role of TF in the treatment of different diseases in patients [9]. In this regard, it should be emphasized that TF can enhance the existing cell-mediated immune responses and affect the activity of various immune components. In addition, it induces immune, rapid, and sensitive responses to the recipients that could be demonstrated within 24-48 h [9]. The caprine serum-derived TF improved the ability to survive high-risk infectious challenges by altering the mice's cytokine response profile [10]. In the course of solid tumor therapy, the TF of human promoted the activation of white blood cells, as well as total lymphocytes and their subsets, thereby stimulating the immune response, especially when used for 12 months in patients [11]. Pineda et al. reported that TF in the adjuvant immunotherapy of experimental glioma, can obviously reduce the tumour size, increased $\mathrm{CD} 2+, \mathrm{CD} 4+, \mathrm{CD} 8+$ and $\mathrm{NK}$ cell counts, it also increased the percentage of apoptotic tumour cells [12]. In Allergy therapy, Transfer factor may restore the Th1/Th2 balance and improve immune regulatory mechanism in patients [13].

In a mouse model infection with larvae cestode Mesocestoides vogae, the TF of human origin enhanced the therapeutic effect of anthelmintic albendazole, reduced serum TGF- $\beta 1$ and IL-17 levels, and regulated Th1/Th2 immune-related cytokines [14]. 
Although the abovementioned results strongly indicate an association between TF and immunotherapy, the effect of TF on intestinal mucosal immunity remains to be explored. Therefore, the present study aimed to explore the effect of TF on intestinal mucosal immunity and related signaling pathways. Moreover, it was attempted to provide a scientific theoretical basis for its application in poultry breeding and treatment.

\section{Materials and Methods}

\section{TF}

Chicken spleen transfer factor was prepared by Jian $\mathrm{Mu}$ Biopharmaceutical Co., Ltd., in which effective concentration was 1 $\mathrm{mg} / \mathrm{mL}$, nucleic acids are $300 \mu \mathrm{g} / \mathrm{mL}$, and the purity is $95 \%$.

\section{Ethics approval and consent to participate}

The study protocol and all animal procedures were approved by the China Agricultural University Institutional Animal Care and Use Committee (AW11050202-2).

\section{Animals and experimental design}

A total of 100 laying hens (one-day-old) were raised under the standard conditions. Water and feed were provided ad libitum. The illumination time was $19 \mathrm{~h}$ at 5 days of age and then decreased by $2 \mathrm{~h}$ every day until it reached $8 \mathrm{~h}$. The ambient temperature was kept at approximately $28-30^{\circ} \mathrm{C}$ during 4-7 days, which was then reduced by $2^{\circ} \mathrm{C}$ every week until it reached $18-20^{\circ} \mathrm{C}$. The animals were randomly divided into a control group and experimental groups (20 laying hens per group) treated with different doses of TF $0.05 \mathrm{~mL}, 0.10 \mathrm{~mL}, 0.25 \mathrm{~mL}$, and $1.00 \mathrm{~mL}$ via oral administration from the $5^{\text {th }}$ day to the $12^{\text {th }}$ day. Immediately, the tissues were harvested on the $12^{\text {th }}$ day $(n=10)$ or continued to raise a week without TF. Subsequently, the tissues were harvested on the $19^{\text {th }}$ day $(n=10)$. The experimental animals were weighed and euthanized under anesthesia using $10 \%$ chloral hydrate. Following that, the samples of the duodenum, jejunum, ileum, and rectal tissues were collected and divided into two parts. One part of the intestinal tissue was fixed in $4 \%$ paraformaldehyde for histologic observation, and the other part was quickly frozen in liquid nitrogen for protein or RNA extraction. Furthermore, the whole lymphoid organs (splenic, thymus, and bursa) were harvested and weighed to record the organ index (Organ index = organ weight $(\mathrm{g})$ /body weight (g) $\times 100 \%)$.

\section{Intestinal histological analysis}

Small intestines samples $(n=6)$ were immediately fixed in $4 \%$ paraformaldehyde in $0.1 \mathrm{M}$ phosphate-buffered saline for $48 \mathrm{~h}$ and embedded in paraffin for sectioning $(5 \mu \mathrm{m}$, cross-section), then stained with haematoxylin and eosin (H\&E) and periodic acid-Schiff (PAS). For H\&E staining, at least 10 tissue sections were cut from each sample and photographed at 400×magnifications using a BX51 microscope (Olympus, Tokyo, Japan). Five longest villi in each tissue section and a total of 300 longest villi were analysed in each treatment group. Subsequently, Image-Pro Plus 6.0 (Media Cybernetics, USA) software was used to measure the Villus Height (VH), Crypt Depth (CD) and the $\mathrm{V} / \mathrm{C}$ ratio. The $\mathrm{VH}$ and $\mathrm{CD}$ were measured from the opening of the intestinal gland to the top of the villus and muscularis mucosa, respectively. The number of intraepithelial lymphocyte per 100 enterocytes was calculated by Image-Pro Plus 6.0 software. For the analysis of PAS-staining, at least 30 random fields in 6 sections were photographed for each sample, and a total of 180 fields were analysed per treatment group. The number of goblet cells per 100 enterocytes was calculated.

\section{Enzyme-linked immunosorbent assay}

Prior to Enzyme-Linked Immunosorbent Assay (ELISA) analysis, the total protein concentrations of the intestinal tissue lysates were determined using a Bicinchoninic Acid (BCA) assay kit (CW0014, Beijing Co Win Biotech Co., Ltd., Beijing, China). The detection range for this assay was from 20 to $2000 \mu \mathrm{g} / \mathrm{mL}$. The IL-10 and TNF- $\alpha$ levels in the intestinal tissues were measured using a biotin labeling double-antibody sandwich ELISA (LBTR-EL-1648, LBTR-EL-1643, Beijing Limbo Terry Technology Development co., Ltd., Beijing, China). Detection ranges for IL-10 and TNF- $\alpha$ were $5 \mathrm{pg} / \mathrm{mL} \sim 1500 \mathrm{pg} /$ $\mathrm{mL}$ and $5 \mathrm{ng} / \mathrm{L} \sim 1000 \mathrm{ngL}$, respectively. Six samples were used in each group, with each sample tested in triplicate. All tests were performed according to the manufacturer's instructions. Results were quantified by measuring Optical Density (OD) at $450 \mathrm{~nm}$ wavelength. The intraand inter-assay variations were $<10 \%$ and $<12 \%$, respectively.

\section{Total RNA extraction, reverse transcription, and quantitative real-time PCR}

Total RNA was extracted from duodenum, jejunum, ileum, and rectal sections of each treatment $(\mathrm{n}=6)$ using a Trizol Reagent (CW0580A, Beijing Co Win Biotech Co., Ltd., Beijing, China) following the manufacturer's protocol. The mRNA was then reverse transcribed to cDNA using a GoScript ${ }^{\mathrm{TM}}$ Reverse Transcription System (A5000, Promega, USA). The cDNA was used as a template for quantitative real-time PCR analysis, and the sequences for Muc2, TLR-2, and TLR-4 primers were obtained from GenBank (Table 1). The RT-qPCR was performed in a $10 \mu \mathrm{L}$ mixture containing $5 \mu \mathrm{L}$ Go Taq Green Master Mix (Promega, USA), $0.1 \mu \mathrm{L}$ of forward, $0.1 \mu \mathrm{L}$ of reverse primer, $3.8 \mu \mathrm{L}$ RNAase-free water, and $1 \mu \mathrm{L}$ cDNA. The PCR reaction conditions were as follows: denaturation at $94^{\circ} \mathrm{C}$ for $30 \mathrm{~s}$, annealing at $56^{\circ} \mathrm{C}$ for $30 \mathrm{~s}$, and extension at $72^{\circ} \mathrm{C}$ for $60 \mathrm{~s}$. PCR products were electrophoresis in a $2 \%$ agarose gel containing Ethyl Ingot Bromide (EB), The results were analyzed using a GelPro Analyzer v4.0 (Media Cybernetics, USA), and each sample was assayed in triplicate.

\section{Measurements of antioxidant activity}

Portions of the intestinal segments $(n=6)$ were rapidly homogenized and clarified lysates were obtained by centrifugation at $200 \times \mathrm{g}$ for $10 \mathrm{~min}$ at $4^{\circ} \mathrm{C}$. Tissue extracts were stored at $80^{\circ} \mathrm{C}$ prior to the analysis of antioxidant activity. Protein concentrations of the intestinal tissue lysates were determined using a BCA assay kit (CW0014, Beijing Co Win Biotech Co., Ltd., Beijing, China). Reactive Oxygen Species (ROS) assay kit (CA1410, Beijing Solarbio Science \& Technology Co., Ltd., Beijing, China) and five commercial kits (Nanjing Jiancheng Co. Ltd., Jiancheng, Nanjing, China) were used to assess antioxidant ability, the activities of Superoxide Dismutase (SOD), Catalase (CAT), Glutathione Peroxidase (GSH$\mathrm{Px}$ ) activities, as well as Total Antioxidant Capability (T-AOC), and Malondialdehyde (MDA) levels were quantified using colorimetric methods. SOD, CAT, GSH-Px, T-AOC, and MDA were measured as $\mathrm{OD}$ at $550 \mathrm{~nm}, 405 \mathrm{~nm}, 412 \mathrm{~nm}, 520 \mathrm{~nm}$, and $532 \mathrm{~nm}$ wavelengths, respectively. SOD, CAT, and GSH-Px activities were expressed as specific activity (units/mg protein), while T-AOC and MDA were 
Table 1: Primers used for quantitative real-time PCR analysis.

\begin{tabular}{|c|c|c|c|c|c|}
\hline & Primer Sequences (5'-3') & Accession no. & Product Size (bp) & $\operatorname{Tm}\left({ }^{\circ} \mathrm{C}\right)$ & Cycles \\
\hline \multirow{2}{*}{ Muc2 } & F: 5'-CTACTTCACCTTCAACCATTACAACG-3' & \multirow{2}{*}{ XM_421035.2 } & \multirow{2}{*}{163} & \multirow{2}{*}{56} & \multirow{2}{*}{29} \\
\hline & R: 5'-TCATAGTCACCACCATCTTCTTCAG-3' & & & & \\
\hline \multirow{2}{*}{$T L R-2$} & F: 5'-AGGCACTTGAGATGGAGCAC-3' & \multirow{2}{*}{ AB046533 } & \multirow{2}{*}{287} & \multirow{2}{*}{55} & \multirow{2}{*}{30} \\
\hline & R: 5'-CCTGTTATGGGCCAGGTTTA-3' & & & & \\
\hline \multirow{2}{*}{$T L R-4$} & F: 5'-GGCTCAACCTCACGTTGGTA-3' & \multirow{2}{*}{ NM_001030693.1 } & \multirow{2}{*}{220} & \multirow{2}{*}{56} & \multirow{2}{*}{30} \\
\hline & R: 5'-AGTCCGTTCTGAAATGCCGT-3' & & & & \\
\hline \multirow{2}{*}{ Gapdh } & F: 5'-ATCACAGCCACACAGAAGACG-3' & \multirow{2}{*}{ NM_204305.1 } & \multirow{2}{*}{124} & \multirow{2}{*}{56} & \multirow{2}{*}{25} \\
\hline & R: 5'-TGACTTTCCCCACAGCCTTA-3' & & & & \\
\hline
\end{tabular}

Note: Sequences for primers of Muc2, TLR-2 and TLR-4 were obtained from Genbank and NCBI. For qRT-PCR reactions, $10 \mu L$ mixtures were made by using $5 \mu L$ GoTaq Green Master Mix (Promega, USA), $0.1 \mu \mathrm{L}$ of forward and $0.1 \mu \mathrm{L}$ of reverse primer, 3.8 $\mu \mathrm{L}$ RNAase-free water and $1 \mu \mathrm{L}$ cDNA. The PCR conditions were available upon request. Results were analyzed using gel-pro Analyzer v4.0 (Media Cybernetics, USA). Each sample was assayed three times. Abbreviations: F: Forward; R: Reward.

expressed as units/mg protein and $\mathrm{mmol} / \mathrm{mg}$ protein, respectively. Each sample was assayed three times. Intra- and inter-assay variations were determined to be $<10 \%$.

\section{Statistical analysis}

The data were analyzed in SPSS software (version 2.0) (SPSS Inc., USA) and represented as mean $\pm \mathrm{SD}$. One-way ANOVA was used to analyze the significance of differences between groups using LSD as the standard. Different lowercase letters represent significant differences between groups. $\mathrm{p}<0.05$ was considered statistically significant.

\section{Results and Discussion}

Peptides or proteins is a relatively new weapon against severe infections caused by multi-drug resistant bacteria. Currently, the Food and Drug Administration has approved nearly 380 peptides and proteins on the market, which will make up an even larger proportion of the market in the future [15]. The TF is a low molecular weight lymphocyte extract that can nonspecifically enhance the immune function of the recipient animals [8]. This study aimed to investigate the effects of different doses of TF on intestinal digestion, absorption function, and intestinal mucosal barrier in laying hens.

Although the oral administration of TF tends to an increase in the weight and the index of immune organs, there was no significant difference between experimental and control groups in this regard (Figure 1a-1c), this may be due to the short application time, or the effect of feed additives on the weight gain of laying hens is not obvious.

As an important part of the small intestine, the morphological changes of intestinal villi also directly affect the surface area of the intestinal villi, and then affect the ability of the body to absorb nutrients [16].

The ratio of villus height to crypt depth represents the absorption capacity of small intestine [17-19]. The results showed that after treatment with different doses of TF for a week, the VH and V/C from the duodenum to ileum sections increased significantly, especially in the TF- $0.25 \mathrm{~mL}$ and TF- $1.00 \mathrm{~mL}$ group, however, a significant decrease was observed in the CD (Figure 2b, 2d and 2f). After the treatment termination with TF for one week, the VH, $\mathrm{CD}$, and $\mathrm{V} / \mathrm{C}$ maintained the same trend in $\mathrm{TF}-0.25 \mathrm{~mL}$ and $\mathrm{TF}-1.00 \mathrm{~mL}$ groups (Figure

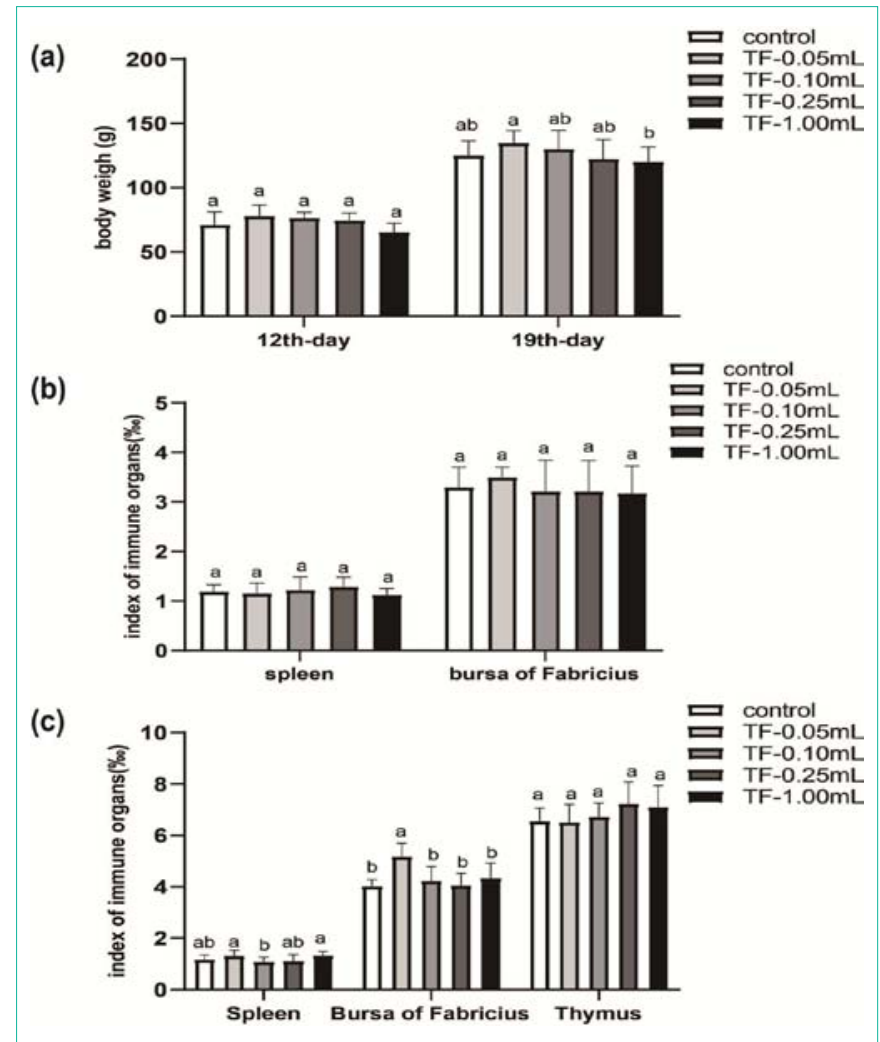

Figure 1: Effect of TF on the body weight (a) and the immune organs index (b or c) in 12d and 19d. The values are presented as the means \pm SD $(n=10)$. Mean values without the same marker $(a, b)$ represent statistically significant differences $(p<0.05)$.

$2 \mathrm{c}, 2 \mathrm{e}$ and $2 \mathrm{~g}$ ). It indicates that TF has an influence on intestinal mucosa morphology and improves intestinal mucosa digestion and absorption, which may be related to the active ingredient in TF. Studies have shown that antibacterial peptides can significantly increase the ratio of the jejunum and ileum $\mathrm{VH} / \mathrm{CD}$, jejunal $\mathrm{CD}$, and ileal $\mathrm{VH}[5]$.

Fungus-derived macromolecules, including nucleotides, peptides, and glutamic acid can enhance the gut health in chickens, promote intestinal epithelial cell growth, and regulate immunity [20]. Furthermore, nucleotides can promote the development of intestinal cells in weaned rats, promote growth and maturation, and improve 

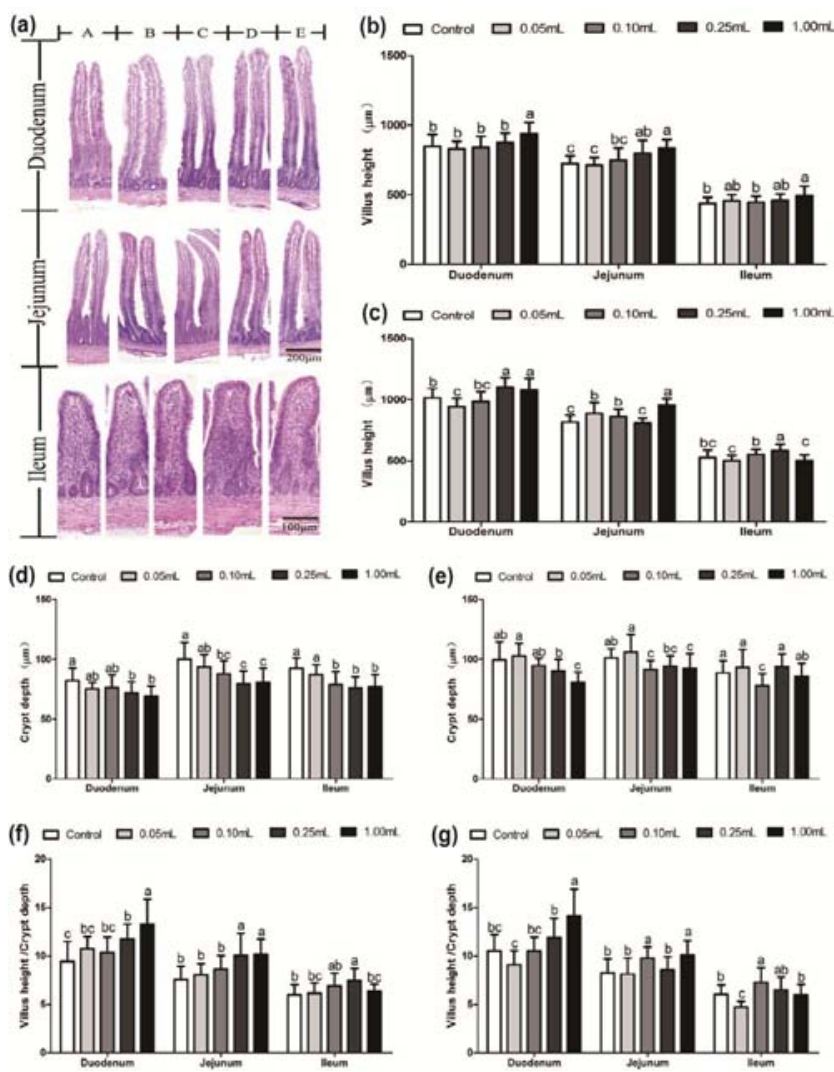

Figure 2: The morphology of intestine in each group was analyzed using HE staining. The duodenum, jejunum and ileum of different treatment chicken in 12d. A) Normal control group; B) TF-0.05mL group; C) TF-0.10mL group; D) TF-0.25mL group; E) TF-1.00mL group. Scale bar $200 \mu \mathrm{m}$ in the duodenum, jejunum, Scale bar 100um in the ileum. Effect of TF on the villus height of small intestine of laying hens in $12 d$ (b) or $19 d$ (c). Effect of TF on the crypt depth of small intestine of laying hens in $12 d(d)$ or $19 d(e)$. Effect of TF on the villus height /crypt depth of small intestine of laying hens in 12d (f) or 19d (g). The values are presented as the means $\pm S D(n=10)$. Mean values without the same marker $(a, b)$ represent statistically significant differences $(p<0.05)$.

intestinal mucosal morphology [21]. Adding cecropin AD to the diet can stimulate the intestinal mucosa to produce more secretory immunoglobulin A, which can activate the body and local immune system to cope with the attack of $E$. coli, and subsequently improves piglet performance [5].

According to the above studies, it is found that TF can effectively improve the intestinal mucosal morphological structure. Additionally, the TF activates the immune system [22], accordingly, its effect on intestinal mucosal immunity is very worthy of discussion. The number of Intraepithelial Lymphocyte (IELs) was analyzed, and the contents of IL-10, as well as TNF- $\alpha$, were detected by ELISA. The results showed that continuous administration of TF to laying hens one week could significantly increase the number of intraepithelial lymphocytes and IL-10 content in each intestinal segment, thereby reducing the TNF- $\alpha$ content significantly (Figure $3 b, 3 d$ and $3 \mathrm{f}$ ). In addition, a treatment cessation for one week had a significant effect on laying hens (Figure 3c, $3 \mathrm{e}$ and $3 \mathrm{~g}$ ). A similar finding has been reported in healthy mice that were fed with low-fat egg yolk which improved immunity; moreover, the egg yolk low lipid peptic digests increased IL-10and IL-4 expression, as well as macrophage phagocytosis [23].
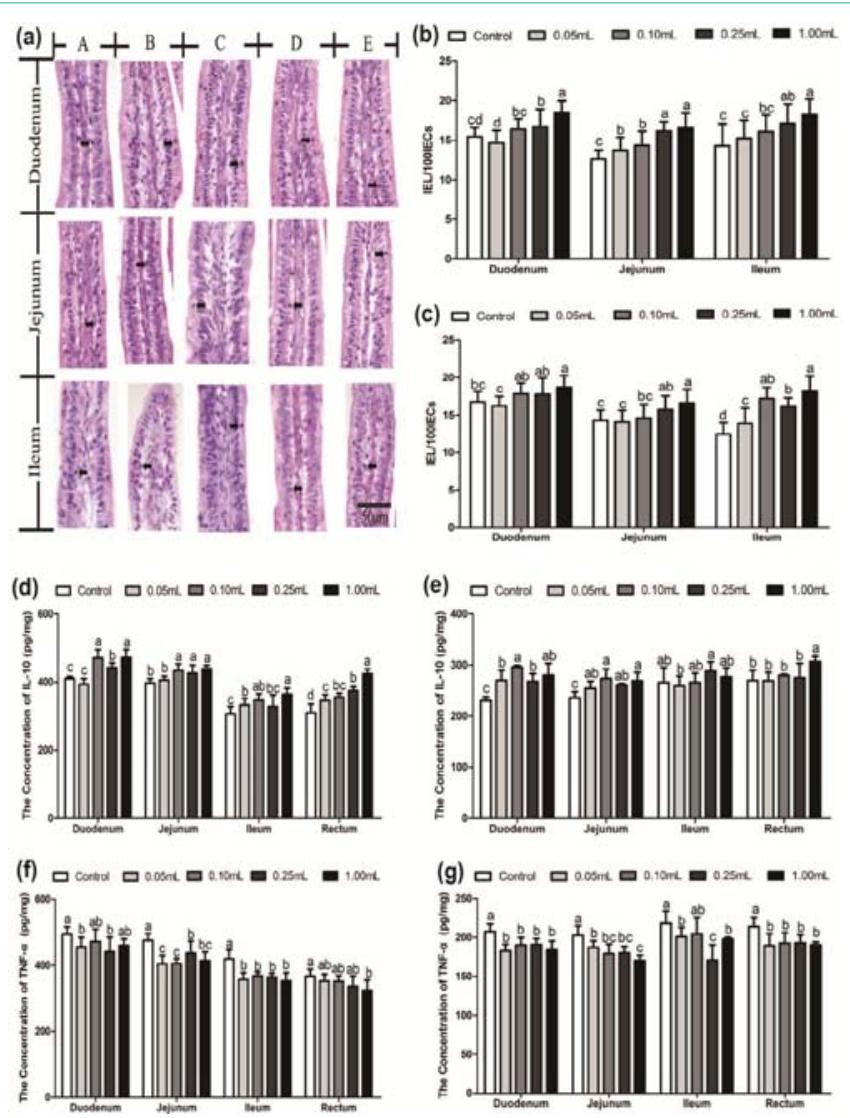

Figure 3: The distribution of IEL in intestine of different treatment laying hens in $12 \mathrm{~d}$ (HE staining). A) Normal control group; B) TF-0.05mL group; C) TF$0.10 \mathrm{~mL}$ group; D) TF-0.25mL group; E) TF-1.00mL group; Scale bar=50 $\mu \mathrm{m}$. Effect of TF on the IEL/100IECs of small intestine of laying hens in $12 \mathrm{~d}$ (b) or $19 \mathrm{~d}$ (c). Effect of TF on the concentration of IL-10 of small intestine and rectum of laying hens in $12 d(d)$ or $19 d(e)$. Effect of TF on the TNF-a concentration of small intestine and rectum of laying hens in $12 d(f)$ or $19 d(g)$. The values are presented as the means $\pm S D(n=10)$. Mean values without the same marker $(a, b)$ represent statistically significant differences $(p<0.05)$.

The use of fermented milk as food significantly enhances the production of IL- 6 by intestinal epithelial cells and IgA, thereby improving the intestinal immune defense and increasing the host protection against pathologies [24]. Active peptides stimulate the up-regulation of immunity in healthy animals, and studies have shown that continuous feeding of three weeks of bioactive peptides in mice can effectively alleviate the pathological response to LPS. Furthermore, according to the results, bioactive peptides increased circulating anti-inflammatory factors IL-10 and IL- 4 and inhibited TNF- $\alpha$ associated inflammatory responses [25].

Zhang et al. [26] designed a hybrid peptide effectively to prevent LPS-induced mucosal barrier damage and intestinal inflammation. The obtained results are consistent with the findings in this study. The TF improved the intestinal mucosal immune function with an increase in the number of IELs and content of IL-10, followed by a decrease in the content of TNF- $\alpha$.

The number of goblet cells was analyzed by PAS staining, and the Muc2 expression was detected by the RT-PCR. The results showed that continuous or discontinuous feeding of TF for a week could significantly increase the number of goblet cells and up-regulate the 

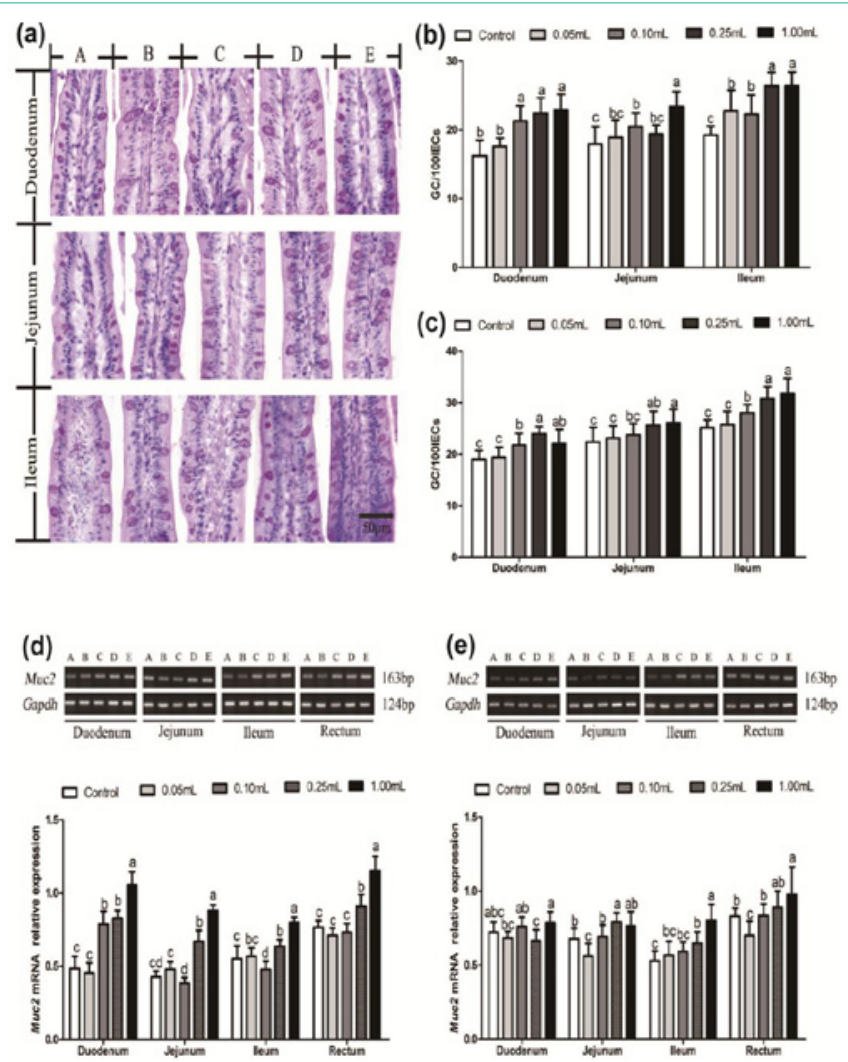

Figure 4: The distribution of goblet cells in intestine of different treatment laying hens in 12d (PAS staining). A) Normal control group; B) TF-0.05mL group; C) TF-0.10mL group; D) TF-0.25mL group; E) TF-1.00mL group. Bar=50um. Effect of TF on the GC/100IECs of small intestine of laying hens $12 d(b)$ or $19 d(c)$. Electrophoretogram and relative Muc2 mRNA expression level in small intestine and rectum in $12 \mathrm{~d}$ (d) or $19 \mathrm{~d}(\mathrm{e})$. The values are presented as the means $\pm \operatorname{SD}(n=10)$. Mean values without the same marker $(a, b)$ represent statistically significant differences $(p<0.05)$.

relative expression of Muc2 gene (Figure 4b-4e).

Studies have shown that the mouse Cathelin-Related Antimicrobial Peptide (mCRAMP) reversed the decrease of colonic mucus thickness in colitis through the upregulation the expression of mucin genes (MUC1, MUC2, MUC3 and MUC4) in colonic tissues [27].

Plaisancié et al. [27] demonstrated that the peptide $\beta-\mathrm{CN}$ (94-123) present in yoghurts enhanced the number of goblet cells, stimulated MUC2 and MUC4 gene expression, maintained and repaired the balance of the intestinal environment, and protected the intestine from the microbial damage of the intestinal cavity, which was in line with the results of the current study.

The findings obtained from the present study revealed that TF could effectively improve the intestinal IL-10 content. Moreover, one study found that IL-10 had a direct effect on goblet cells to regulate the synthesis and secretion of mucin. Similarly, a significantly decreased MUC2 production has been demonstrated in IL-10-deficient mice [28]. Therefore, it seems that the TF might cause pluripotent stem cells to differentiate from the goblet cells by the up-regulation of IL10 to increase the number of goblet cells and the relative expression of the Muc2 gene.
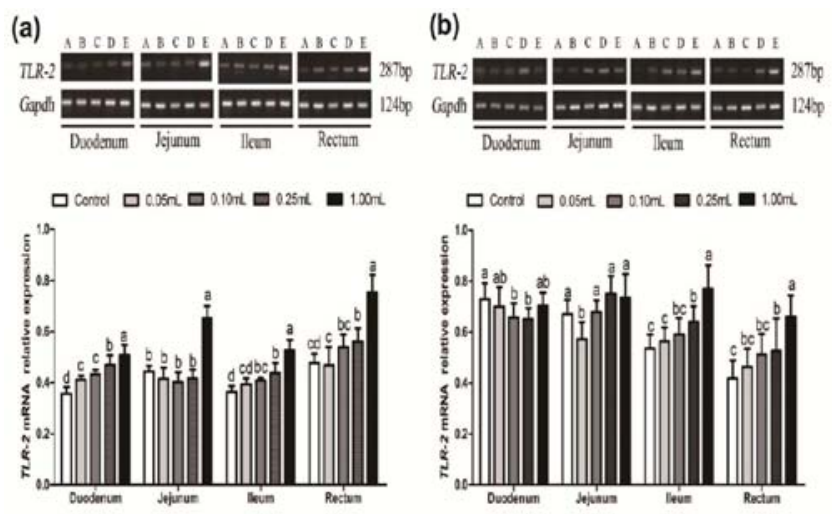

(c)

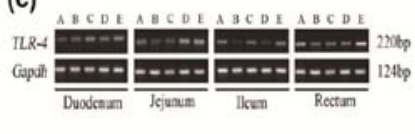

(d)
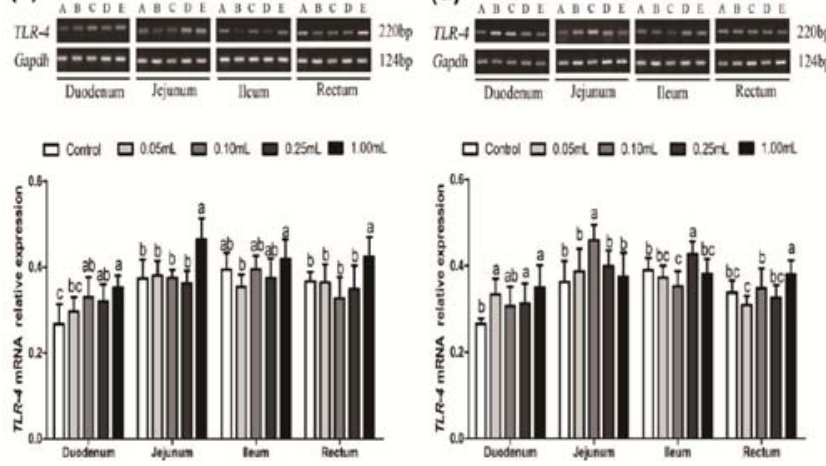

Figure 5: Electrophoretogram and relative TLR-2 mRNA expression level in small intestine and rectum of laying hens in $12 d$ (a) or 19d (b). Electrophoretogram and relative TLR-4 mRNA expression level in small intestine and rectum of laying hens in $12 \mathrm{~d}$ (c) or $19 \mathrm{~d}$ (d). A) Normal control group; B) TF-0.05mL group; C) TF-0.10mL group; D) TF-0.25mL group; E) TF-1.00mL group. The values are presented as the means \pm SD $(n=10)$ Mean values without the same marker $(a, b)$ represent statistically significant differences $(p<0.05)$.

According to a study conducted by Jensen, a variety of intestinal inflammatory diseases were associated with abnormally elevated levels of TLR-2 and TLR-4 [29]. However, studies have found that dietary $\mathrm{NiCl} 2$ reduced the expression of TLR2 (TLR2-2) and TLR4 mRNA in intestinal mucosa, damaged the innate immune system of intestinal mucosa, thereby influencing the intestinal homeostasis [5]. Accordingly, it is hypothesized that the decreased expression of TLR2 and TLR- 4 may cause intestinal mucosal damage, decrease cytokine secretion, and impair intestinal immune function. To explore the regulation role of TF in the intestinal mucosal immunity, the expression of toll like receptor (TLR-2 and TLR-4) was detected by the RT-PCR. The results of this study demonstrated that continuous administration of TF to laying hens increased the relative expression of TLR-2 and TLR-4 genes in the intestine, and the expression level remained elevated after one week of withdrawal (Figure 5a-5d). The above studies have shown that TF significantly increases intestinal IL-10 levels, and TLR2 signals promote a rapid induction and degrade the IL-10. However, the TLR4 stimulation of BMMs leads to higher levels of IL-10 production, which may be beneficial to inhibit inflammatory pathologies [30]. The IL-10 inhibits inflammationinduced Endoplasmic Reticulum (ER) stress response mechanisms by modulating ATF-6 transcriptional activity [31]. MyD88 is an adaptor 


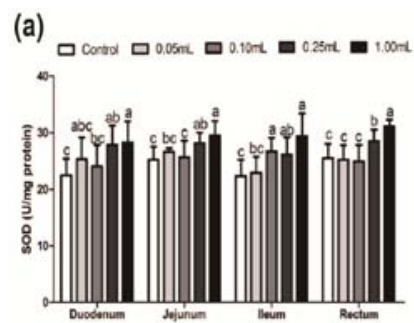

(c)

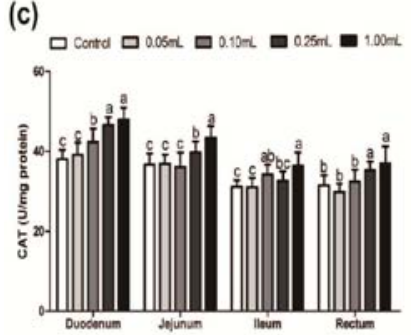

(b)

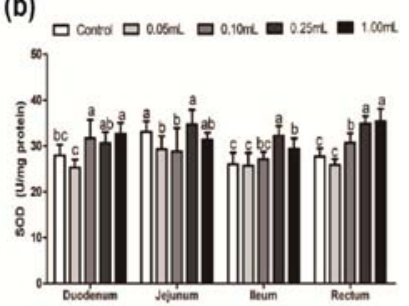

(d)

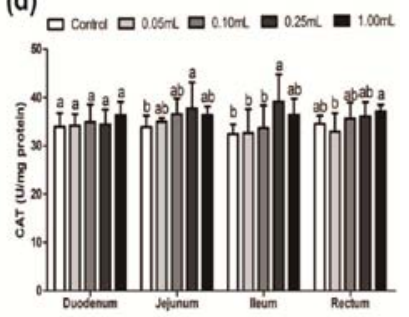

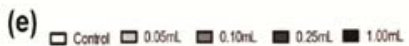
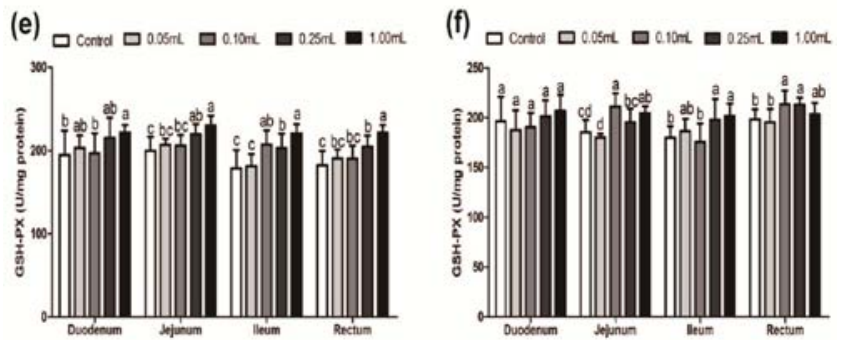

Figure 6: Effect of TF on the content of the antioxidant enzymes SOD (a or b), CAT (c or d), GSH-PX (e or f), the total antioxidant capacity of T-AOC ( $g$ or $h$ ) and the lipid peroxidation products of MDA ( $i$ or j) in small intestine and rectum in $12 \mathrm{~d}$ or $19 \mathrm{~d}$. The values are presented as the means \pm SD $(n=10)$. Mean values without the same marker $(a, b)$ represent statistically significant differences $(p<0.05)$

molecule in signal transduction by TLR [32]; moreover, MyD88dependent TLR signal plays a critical role in host defense against an enteric bacterial pathogen.

Gibson et al. [33] and Frantz et al. [34] reported that the defect in epithelial barrier function in MyD88 mice may have reduced the expression of antimicrobial peptides, MUC2, and TLRs. This also indicates that TLRs are closely related to the production of MUC2. Consequently, it is hypothesized that the up-regulation of TLR expression may also be the cause of the up-regulation of Muc2 expression.

Although there are complex antioxidant systems in the body to maintain the body's oxidative balance, oxidative stress occurs when the production of reactive oxygen species exceeds the scavenging capacity of the antioxidant system [35]. Antioxidant enzymes (i.e., SOD, CAT, and GSH-PX), total antioxidant capacity of T-AOC, and lipid peroxidation products of MDA were detected to explore the effect of TF on the antioxidant function of the intestine. The results showed that continuous administration of TF to laying hens could significantly increase the various antioxidant enzymes SOD and CAT in each intestinal, as well as the total antioxidant capacity of T-AOC, which could significantly reduce the content of malondialdehyde MDA lipid peroxidation products (Figure $6 a, 6 c$ and $6 e$ ). After the treatment termination with $\mathrm{TF}$, the effect of $\mathrm{TF}$ on intestinal
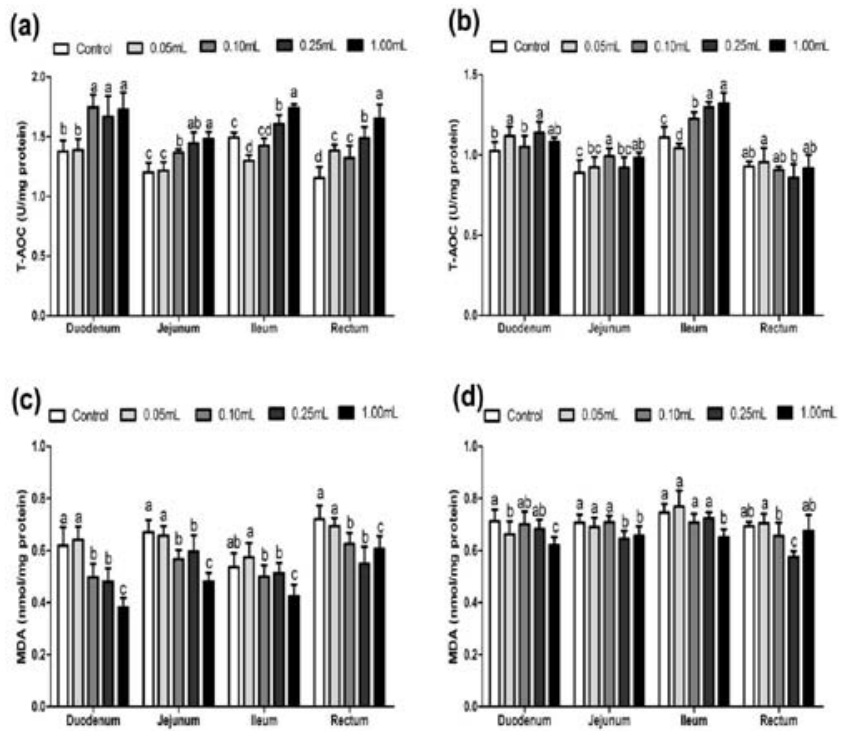

Figure 7: Effect of TF on the total antioxidant capacity of T-AOC ( $a$ or b) and the lipid peroxidation products of MDA (c or d) in small intestine and rectum in $12 d$ or $19 d$. The values are presented as the means $\pm S D(n=10)$. Mean values without the same marker $(a, b)$ represent statistically significant differences $(p<0.05)$.

antioxidant function was weakened (Figure 6b, 6d and 6f).

Accordingly, it can be hypothesized that TF can significantly improve intestinal antioxidant capacity probably due to $\mathrm{TF}$ containing biologically active peptides or amino acids. In this regard, studies have shown that amino acids have protective effects against tissue oxidative stress in intestinal epithelial cells based on the structure [36]. Dietary supplementation of Sulfur Amino Acids (SAA) can improve the intestinal antioxidant capacity of weaned piglets [37]. Hong reported that wheat peptide could significantly attenuated the activities of superoxide dismutase and glutathione peroxidase induced by indomethacin, thereby increasing the activities of antioxidant enzymes in IEC-6 cells [38]. Toll-like Receptors (TLRs) induce inflammation and tissue repair through multiple signaling pathways [39]. The mounting evidence suggests that the Nrf2 pathway plays a key role in tissue damage caused by microbial infections or inflammation-related diseases; additionally, the TLR signaling regulated the Nrf2 signaling pathway [40]. The Nrf2 is a key transcription factor that mainly regulates cellular defenses against oxidative stress. It is combined with antioxidant-related components (ARE) to promote the production of related antioxidants [41]. This result is consistent with the findings in this study in which it was found that the oral administration of TF significantly increased the expression of intestinal TLR-2 and TLR-4.

Therefore, it is hypothesized that TF regulates the intestinal mucosal immune system by binding to TLR- 2 or TLR- 4 receptors, increases the number of intraepithelial lymphocytes and the content of anti-inflammatory factor IL-10 in the intestinal tract, decreases the content of inflammatory factor TNF- $\alpha$. IL-10, promotes the differentiation of pluripotent stem cells into goblet cells to increase the number of goblet cells, up-regulates the relative expression of the Muc2 gene to improve intestinal mucus barrier, and activates 
the TLR signaling pathway to activate Nrf2 binding to ARE, which is a key element of antioxidant reaction to improve the antioxidant function of the body.

\section{Conclusion}

In conclusion, high-dose (TF-1.00mL) of TF can be administered to laying hens for 7 consecutive days to improve the intestinal mucosa morphology and structure, enhance digestion and absorption functions, enhance the intestinal mucosal barrier immune function and antioxidant function, and up-regulate Muc2, TLR-2 and TLR-4 gene relative expression. The effect of TF on the intestinal mucosal barrier can be sustained after one week of drug withdrawal, but the effect has weakened. By exploring the effect of TF on intestinal mucosal immunity and related signaling pathways, it is helpful reveal the mechanism of TF to strengthen the intestinal mucosal immune barrier and to provide scientific theoretical basis for its application in poultry breeding and resistance pathogen infection.

\section{References}

1. Antosova Z, Mackova M, Kral V, Macek T. Therapeutic application of peptides and proteins: parenteral forever? Trends Biotechnol. 2009; 27: 628-635.

2. Singh $S$, et al. PEPstrMOD: structure prediction of peptides containing natural, non-natural and modified residues. Biol Direct. 2015; 10: 73.

3. Erak $\mathrm{M}$, et al. Peptide chemistry toolbox-Transforming natural peptides into peptide therapeutics. Bioorg Med Chem. 2018; 26: 2759-2765.

4. Fjell CD, Hiss JA, Hancock REW, Schneider G. Designing antimicrobial peptides: form follows function. Nat Rev Drug Discov. 2011; 11: 37-51.

5. Wu S, et al. Effects of the antimicrobial peptide cecropin AD on performance and intestinal health in weaned piglets challenged with Escherichia coli. Peptides. 2012; 35: 225-230.

6. Fasina YO, Thanissery RR. Comparative efficacy of a yeast product and bacitracin methylene disalicylate in enhancing early growth and intestinal maturation in broiler chicks from breeder hens of different ages. Poult. Sci. 2011; 90: 1067-1073.

7. Superchi $P$, et al. Effects of dietary nucleotide supplementation on growth performance and hormonal and immune responses of piglets. Animal. 2012; 6: $902-908$.

8. Dwyer JM. Transfer factor in the age of molecular biology: a review. Biotherapy. 1996; 9: 7-11

9. Viza D, et al. Transfer factor: an overlooked potential for the prevention and treatment of infectious diseases. Folia Biol (Praha). 2013; 59: 53-67.

10. Willeford BV, Shapiro-Dunlap T, Willeford KO. Serum Derived Transfer Factor Stimulates the Innate Immune System to Improve Survival Traits in HighRisk Pathogen Scenarios. Drug Dev Res. 2017; 78(5): 189-195.

11. Garritano CRO, Nubila FD, Couto RM, Fiorelli RKA, Aun LB. Use of transfer factor in immunosuppressed surgical patients. Rev Col Bras Cir. 2017; 44: 452-456.

12. Pineda B, et al. Interstitial transfer factor as adjuvant immunotherapy for experimental glioma. J Exp Clin Cancer Res. 2005; 24: 575-583.

13. Vera JG, Sánchez RC, Sandoval GF, Solano MO, Parra SE. Transfer factor and allergy. Alergia. 2010; 57: 208-214.

14. Kubašková TM, Mudroňová D, Velebný S, Hrčková G. The utilisation of human dialyzable leukocyte extract (IMMODIN) as adjuvant in albendazole therapy on mouse model of larval cestode infection: immunomodulatory and hepatoprotective effects. Int Immunopharmacol. 2018; 65: 148-158.

15. Usmani SS, et al. THPdb: Database of FDA-approved peptide and protein therapeutics. PLoS One. 2017; 12: e0181748.

16. Xing S, et al. Effects of alanyl-glutamine supplementation on the small intestinal mucosa barrier in weaned piglets. Asian-Australas J. Anim. Sci. 2017; 30: 236-245

17. $\mathrm{Li} \mathrm{H}$, et al. Postnatal developmental changes of the small intestinal villus height, crypt depth and hexose transporter mrna expression in supplemental feeding and grazing goats. Small Ruminant Research. 2016; 141: 106-112.

18. Montagne L, Pluske JR. Hampson DJ. A review of interactions between dietary fibre the intestinal mucosa, and their consequences on digestive health in young non-ruminant animals. Anim Feed Sci Technol. 2003; 108: 95-117.

19. Pluske JR, et al. Maintenance of villus height and crypt depth, and enhancement of disaccharide digestion and monosaccharide absorption, in piglets fed on cows' whole milk after weaning. Br J Nutr. 1996; 76: 409-422.

20. Yitbarek A, et al. Performance, histomorphology, and toll-like receptor, chemokine, and cytokine profile locally and systemically in broiler chickens fed diets supplemented with yeast-derived macromolecules. Poult. Sci. 2013; 92: 2299-2231.

21. Lee HJ. Protein Drug Oral Delivery: The Recent Progress. Arch Pharm Res. 2002; 25: 572-584

22. Fabre RA, et al. Transfer factors as immunotherapy and supplement of chemotherapy in experimental pulmonary tuberculosis. Clin Exp Immunol. 2004; 136: 215-223.

23. Nelson R, Katayama S, Mine Y, Duarte J, Matar C. Immunomodulating effects of egg yolk low lipid peptic digests in a murine model. Food Agr Immunol. 2007; 18: 1-15.

24. Vinderola G, Matar C, Palacios J, Perdigón G. Mucosal immunomodulation by the non-bacterial fraction of milk fermented by Lactobacillus helveticus R389. Int J Food Microbiol. 2007; 115: 180-186.

25. Zhou J, et al. Immunomodulating effects of casein-derived peptides QEPVL and QEPV on lymphocytes in vitro and in vivo. Food Funct. 2014; 5: 2061 2069.

26. Zhang L, et al. A Novel Peptide Ameliorates LPS-Induced Intestinal Inflammation and Mucosal Barrier Damage via Its Antioxidant and Antiendotoxin Effects. Int J Mol Sci. 2019; 20: 3974

27. Plaisancié $P$, et al. Immunol A novel bioactive peptide from yoghurts modulates expression of the gel-forming MUC2 mucin as well as population of goblet cells and Paneth cells along the small intestine. J Nutr Biochem. 2013; 24: 213-221.

28. Van der Sluis M, et al. Combined defects in epithelial and immunoregulatory factors exacerbate the pathogenesis of inflammation: mucin 2-interleukin 10-deficient mice. Lab Invest. 2008; 88: 634-642.

29. Frolova L, Drastich $\mathrm{P}$, Rossmann $\mathrm{P}$, Klimesova K, Tlaskalova-Hogenova $\mathrm{H}$. Expression of Toll-like receptor 2 (TLR2), TLR4, and CD14 in biopsy samples of patients with inflammatory bowel diseases: upregulated expression of TLR2 interminal ileum of patients with ulcerative colitis. J Histochem Cytochem. 2008; 56: 267-274.

30. Teixeira-Coelho $M$, et al. Differential post-transcriptional regulation of IL-10 by TLR2 and TLR4-activated macrophages. Eur J Immunol. 2014; 44: 856866.

31. Shkoda A, et al. Interleukin-10 blocked endoplasmic reticulum stress in intestinal epithelial cells: impact on chronic inflammation. Gastroenterology. 2007; 132: 190-207.

32. Medzhitov R, et al. MyD88 is an adaptor protein in the hToll/IL-1 receptor family signaling pathways. Mol Cell. 1998; 2: 253-258.

33. Gibson DL, et al. MyD88 signalling plays a critical role in host defence by controlling pathogen burden and promoting epithelial cell homeostasis during Citrobacter rodentium-induced colitis. Cell Microbiol. 2008; 10: 618-631.

34. Frantz AL, et al. Targeted deletion of MyD88 in intestinal epithelial cells results in compromised antibacterial immunity associated with downregulation of polymeric immunoglobulin receptor, mucin-2, and antibacterial peptides. Mucosal Immunol. 2012; 5: 501-512.

35. Burton GJ, Jauniaux E. Oxidative stress. Best Pract Res Cl Ob. 2011; 25: 
287-299

36. Katayama S, Mine Y. Antioxidative activity of amino acids on tissue oxidative stress in human intestinal epithelial cell model. J Agric Food Chem. 2007; 55(21): 8458-8464

37. Yan S, et al. Dietary sulfur amino acids affect jejunal cell proliferation and functions by affecting antioxidant capacity, $\mathrm{Wnt} / \beta$-catenin, and the mechanistic target of rapamycin signaling pathways in weaning piglets. J Anim Sci. 2018; 96: 5124-5133.

38. Yin $\mathrm{H}$, et al. Protective effect of wheat peptides against indomethacin-induced oxidative stress in IEC-6 cells. Nutrients. 2014; 6: 564-574
39. Sabroe I, Parker LC, Dower SK, Whyte MK. The role of TLR activation in inflammation. J Pathol. 2008; 214: 126-135.

40. Yin S, Cao W. Toll-Like Receptor Signaling Induces Nrf2 Pathway Activation through p62-Triggered Keap1Degradation. Mol Cell Biol. 2015; 35: 26732683.

41. Nguyen T, Nioi P, Pickett CB. The Nrf2-Antioxidant Response Element Signaling Pathway and Its Activation by Oxidative Stress. J Biol Chem. 2009; 284: 13291-13295. 